\title{
Lactato sérico como marcador pronóstico en niños gravemente enfermos: un problema de producción o de clearence?
}

\author{
Blood lactate as prognostic marker in critically ill children: \\ a problem related to production or clearance?
}

\section{Ricardo Ronco*}

La evolución temporal del lactato arterial es ampliamente usada como marcador pronóstico en pacientes con enfermedades críticas, especialmente en la fase de inestabilidad.

En el estudio, publicado por Koliski et al. ${ }^{1}$ en éste número del Jornal de Pediatria, se mide durante las primeras 48 horas, de manera seriada, el lactato arterial en niños que ingresan a la UCI en condición grave. Los niños fueron divididos en grupo $\mathrm{A}$, con lactatos $\geq$ a $18 \mathrm{mg} / \mathrm{dl}(2 \mathrm{mmol} / \mathrm{l})(\mathrm{n}=50), y$ grupo $B$, con $<18 \mathrm{mg} / \mathrm{dl}(\mathrm{n}=25)$.

\section{Vea el artículo relacionado en la página 287}

Existen pocos estudios en niños donde se comparen variables hemodinámicas objetivas o de laboratorio con el examen físico. El estudio actual nos muestra una correlación potente y significativa entre los hallazgos clínicos y los niveles de lactato en las primeras 6 horas, donde un $60 \%$ de los niños con lactatos altos tenía algún signo de hipoperfusión. Sin embargo, después de las 6 horas, no había diferencias entre ambos grupos, y los hallazgos clínicos. Parece que el examen físico es un elemento medianamente sensible en pesquisar la acidosis láctica evidente, aunque su especificidad sea dudosa.

Hubo una diferencia significativa en el nivel de glucosa plasmática al ingreso, con medianas de $181 \mathrm{mg} / \mathrm{dl}$ en el grupo A vs. $128 \mathrm{mg} / \mathrm{dl}$ en el $B(p=0,01)$. Ésta fue la única diferencia en los exámenes de laboratorio. Hay dos explicaciones posibles para esta diferencia. Por un lado, la mayor gravedad de los pacientes del grupo A puede explicar cifras mayores de glicemia pero, por otro, la glucosa es un sustrato que, en condiciones de anaerobiosis, puede facili-

* Jefe UCI Pediátrica Clínica Alemana de Santiago. Profesor, Universidad del Desarrollo, Santiago, Chile.

Forma de citar: Ronco R. Lactato sérico como marcador pronóstico en niños gravemente enfermos: un problema de producción o de clearence? J Pediatr (Rio J). 2005;81:271-2. tar la formación de lactato. Sería interesante observar qué relación existe entre la hiperglicemia, como factor independiente, y el lactato en condiciones de anaerobiosis en otro grupo de pacientes.

Como nos muestra el estudio de la Dra. Koliski, en niños postoperados de cardiopatías congénitas con circulación extracorpórea los niveles iniciales de lactato están altos en la gran mayoría. En este caso, es el mantenimiento de estos niveles en el transcurso del tiempo, y no los valores iniciales, lo que debe hacer sospechar mal pronóstico y producir cambios en nuestra terapia.

En el grupo $A$, se describe una mortalidad del $30 \%(15 / 50)$ y, en el grupo $B$, del $12 \%(3 / 25)(p=0,14)$. En este sentido, los pacientes que fallecieron en las primeras 24 horas presentaban lactatos de ingreso significativamente más altos que los que fallecían luego de las primeras 24 horas (lactato de ingreso de $95 \mathrm{mg} / \mathrm{dl}$ vs. $28 \mathrm{mg} / \mathrm{dl}$ ). Con el uso de regresión logística, el nivel de lactato de $27 \mathrm{mg} / \mathrm{dl}$ ( $3 \mathrm{mmol} / \mathrm{l}$ ) a las 24 horas de evolución fue el de mejor sensibilidad $(55,6 \%)$ y especificidad $(97,2 \%)$ para la predicción de mortalidad.

Lo que no responde este estudio es si el mal pronóstico está dado por la excesiva producción de lactato o por su deficiente utilización.

El nivel de lactato en la sangre arterial está determinado por su producción y su eliminación.

La producción de lactato depende de que el piruvato, formado por la vía glicolítica y por aminoácidos, especialmente la alanina, se transforme a lactato por la enzima láctico deshidrogenasa. La relación láctico/piruvato refleja el potencial de óxido-reducción del citosol. La relación normal es de 10-15:1. En situaciones de hipoperfusión tisular, la relación láctico/piruvato se eleva. Sin embargo, en pacientes críticos el aumento del lactato arterial no se explica solamente por la hipoxia. Por ejemplo, la enzima piruvato deshidrogenasa, responsable de pasar el piruvato 
al ciclo de Krebs, puede estar inhibida por endotoxina. En este caso, el aumento del lactato no será secundario a la hipoxemia, sino al aumento de piruvato y la relación L/P no aumentará ${ }^{2}$. Algunos medicamentos como hipoglicemiantes orales, el etanol, las catecolaminas, los broncodilatadores B2, han demostrado causar aumento del lactato arterial sin que el mecanismo de hipoxia tisular esté implicado. Es probable que existan dos tipos de hiperlactatemia en pacientes estables con significación pronóstica muy diferente.

Hiperlactatemia secundaria a sobreproducción aeróbica, con buen pronóstico o una "verdadera" hiperlactatemia por pobre utilización o mal clearence de lactato, con mal pronóstico. Como se demuestra en el estudio de la Dra. Koliski, el lactato elevado inicialmente fue un buen predictor de muerte sólo en la fase inicial de inestabilidad, primeras 24 horas, y no más tarde, cuando se había logrado una mayor estabilidad. Este hecho coincide con lo publicado por Levy et al. ${ }^{3}$, donde los niveles iniciales de lactato no fueron diferentes en los sobrevivientes o en los que murieron mas allá de las 24 horas. De Backer \& Creteur $^{4}$ reportaron que la hiperlactatemia fue equivalente entre los sobrevivientes y los que fallecieron luego de lograr la estabilización en adultos sépticos. También está descrito que pacientes sépticos con lactato normal, pueden o no desarrollar com- plicaciones, es decir, la normolactatemia puede no servir como valor pronóstico. Otro punto de vista es que ciertos pacientes sépticos mantienen lactatos normales porque su producción disminuida es contrabalanceada por un clearence disminuida.

Por último, el estudio de la Dra. Koliski demuestra que, a pesar de tratarse de una población heterogénea, el lactato alto inicial es un marcador importante de gravedad que, en algunas situaciones, puede ser más específico que las mediciones macro hemodinámicas habituales ${ }^{5}$.

\section{Referencias}

1. Koliski A, Cat I, Giraldi DJ, Cat ML. Lactato sérico como marcador prognóstico em crianças gravemente doentes. J Pediatr (Rio J). 2005; $81: 287-92$

2. Mizock BA. Redox pairs, tissue hypoxia, organ dysfunction and mortality. Crit Care Med. 2000;28:270-2.

3. Levy B, Saudone L. Evolution of lactate/pyruvate and ketone body ratios in the early course of catecholamine-treated septic shock. Crit Care Med. 2000;28:114-19.

4. De Backer D, Creteur J. The hepatoesplachnic area is not a common source of lactate in patients with severe sepsis. Crit Care Med. 2001;29:256-1.

5. Ronco R, Rodríguez J, Castillo A. Intragastric tonometry outcome predictor and monitoring utility in pediatric shock [abstract]. Pediatr Chil J. 1998;69:60-4.

\title{
Monitorização contínua de glicemia: uma arma a ser explorada
}

\author{
Continuous glucose monitoring: a practice that should be explored
}

\section{Durval Damiani*}

\begin{abstract}
Apesar de algumas vozes discordantes, a maioria dos diabetólogos sempre teve a noção de que o controle glicêmico era de vital importância para garantir a integridade de vários tecidos, órgãos e sistemas de nosso organismo. Foi, no entanto, em 1993, quando se concluiu um grande projeto de estudo em pacientes diabéticos, conhecido como Diabetes Control and Complications Trial ou $\mathrm{DCCT}^{1}$, que ficou absolutamente claro que o controle glicêmico no diabetes melito (DM) era uma das mais importantes chaves para se evitarem complicações a médio e longo prazos.

Surgiram vários esquemas de administração de insulina, tentando se aproximar do que o organismo usualmente faz, ou seja, disponibilizar insulina a cada momento que

\footnotetext{
* Professor livre-docente, Unidade de Endocrinologia Pediátrica, Instituto da Criança do Hospital das Clínicas, Universidade de São Paulo (USP), São Paulo, SP.
}

Como citar este artigo: Damiani D. Monitorização contínua de glicemia: uma arma a ser explorada. J Pediatr (Rio J). 2005;81:272-3.
\end{abstract}

ingerimos algum alimento. O fato de a produção ser no local adequado (nas ilhotas pancreáticas que insulinizam, em primeiro lugar, o fígado), no momento exato e na quantidade adequada torna uma tarefa razoavelmente complicada a tentativa de imitar, no paciente diabético, o que ocorre com o indivíduo nãodiabético. Não é, de fato, uma tarefa fácil. Em estudo de monitorização contínua em voluntários normais, verificou-se que as oscilações glicêmicas vão de 46 a 118, demonstrando o vigoroso mecanismo de controle glicêmico exercido para manter um adequado "ambiente metabólico" e evitar complicações decorrentes de glicação protéica ${ }^{2}$. Hoje, empregamos o que se convencionou chamar de Tratamento Intensivo do DM, método em que se procura deixar o paciente insulinizado nas 24 horas do dia e quantidades maiores de insulina de absorção ultra-rápida são administradas nos momentos das refeições. No entanto, tratamento intensivo é sinônimo de monitorização intensiva dos níveis glicêmicos, a não ser que embarquemos num vôo cego, sem instrumentos! 